\title{
Applications of Borel Distribution Series on Analytic Functions
}

\author{
Abbas Kareem Wanas ${ }^{1}$ and Jubran Abdulameer Khuttar ${ }^{2}$ \\ ${ }^{1}$ Department of Mathematics, College of Science, University of Al-Qadisiyah, Iraq \\ e-mail: abbas.kareem.w@qu.edu.iq \\ ${ }^{2}$ Department of Mathematics, College of Science, University of Al-Qadisiyah, Iraq \\ e-mail: jubalaa2012@gmail.com
}

\begin{abstract}
The purpose of the present paper is to determine the necessary and sufficient conditions for the power series $B_{\mu}(z)$ whose coefficients are probabilities of the Borel distribution to be in the family $\mathcal{H}(\lambda, \sigma, \delta, \mu)$ of analytic functions which defined in the open unit disk. We derive a number of important geometric properties, such as, coefficient estimates, integral representation, radii of starlikeness and convexity. Also we discuss the extreme points and neighborhood property for functions belongs to this family.
\end{abstract}

\section{Introduction}

Indicate by $\mathcal{A}$ the family of all functions $f$ of the form

$$
f(z)=z+\sum_{n=2}^{\infty} a_{n} z^{n},
$$

which are analytic and univalent in the open unit disk $U=\{z \in \mathbb{C}:|z|<1\}$.

Also, let $W$ denote the subfamily of $\mathcal{A}$ consisting of functions of the form:

Received: March 7, 2019; Accepted: April 2, 2020

2010 Mathematics Subject Classification: 30C45.

Keywords and phrases: analytic function, Borel distribution, probability, coefficient estimates, integral representation, neighborhood.

Copyright (C) 2020 Abbas Kareem Wanas and Jubran Abdulameer Khuttar. This is an open access article distributed under the Creative Commons Attribution License, which permits unrestricted use, distribution, and reproduction in any medium, provided the original work is properly cited. 


$$
f(z)=z-\sum_{n=2}^{\infty} a_{n} z^{n} \quad\left(a_{n} \geq 0\right) .
$$

The function $f \in W$ is said to be starlike of order $\alpha(0 \leq \alpha<1)$ if it satisfies the condition:

$$
\operatorname{Re}\left\{\frac{z f^{\prime}(z)}{f(z)}\right\}>\alpha \quad(z \in U)
$$

and is said to be convex of order $\alpha(0 \leq \alpha<1)$ if it satisfies the condition:

$$
\operatorname{Re}\left\{1+\frac{z f^{\prime \prime}(z)}{f^{\prime}(z)}\right\}>\alpha \quad(z \in U)
$$

Denote by $S^{*}(\alpha)$ and $C(\alpha)$ the families of starlike and convex functions of order $\alpha$, respectively. These families were introduced and studied by Silverman [7].

The elementary distributions such as the Poisson, the Pascal, the Logarithmic, the Binomial have been partially studied in the Geometric Function Theory from a theoretical point of view (see $[1,2,4,5]$ ).

A discrete random variable $x$ is said to have a Borel distribution if it takes the values $1,2,3, \ldots$ with the probabilities $\frac{e^{-\mu}}{1 !}, \frac{2 \mu e^{-2 \mu}}{2 !}, \frac{9 \mu^{2} e^{-3 \mu}}{3 !}, \ldots$ respectively, where $\mu$ is called the parameter.

$$
P(x=r)=\frac{(\mu r)^{r-1} e^{-\mu r}}{r !}, \quad r=1,2,3, \ldots
$$

Now, we introduce a power series whose coefficients are probabilities of the Borel distribution, that is,

$$
\mathcal{M}(\mu, z)=z+\sum_{n=2}^{\infty} \frac{(\mu(n-1))^{n-2} e^{-\mu(n-1)}}{(n-1) !} z^{n}, \quad z \in U,
$$

where $0 \leq \mu \leq 1$. We note that, by using ratio test we conclude that the radius of convergence of the above power series is infinity. 
We also define the series

$$
\mathcal{N}(\mu, z)=2 z-\mathcal{M}(\mu, z)=z-\sum_{n=2}^{\infty} \frac{(\mu(n-1))^{n-2} e^{-\mu(n-1)}}{(n-1) !} z^{n}, \quad z \in U .
$$

Now, we consider the linear operator $B_{\mu}(z): \mathcal{A} \rightarrow \mathcal{A}$ defined by the convolution or Hadamard product

$$
B_{\mu}(z)=\mathcal{N}(\mu, z) * f(z)=z-\sum_{n=2}^{\infty} \frac{(\mu(n-1))^{n-2} e^{-\mu(n-1)}}{(n-1) !} a_{n} z^{n}, \quad\left(a_{n} \geq 0, z \in U\right)
$$

\section{Main Results}

We begin this section by defining the family $\mathcal{H}(\gamma, \delta, \tau, \mu)$ as follows:

Definition 2.1. A function $B_{\mu}$ is said to be in the family $\mathcal{H}(\lambda, \sigma, \delta, \mu)$ if it satisfies

$$
\left|z B_{\mu}^{\prime \prime}(z)\right|<\delta\left|\lambda z B_{\mu}^{\prime \prime}(z)+(\lambda+1)(1-\sigma) B_{\mu}^{\prime}(z)\right|,
$$

where $0 \leq \lambda<1,0 \leq \sigma<1,0<\delta \leq 1,0 \leq \mu \leq 1$ and $z \in U$.

In the first theorem, we establish the necessary and sufficient conditions for the power series $B_{\mu}$ to be in the family $\mathcal{H}(\lambda, \sigma, \delta, \mu)$.

Theorem 2.1. A function $B_{\mu}$ is in the family $\mathcal{H}(\lambda, \sigma, \delta, \mu)$ if and only if

$$
\sum_{n=2}^{\infty} \frac{n(\mu(n-1))^{n-2} e^{-\mu(n-1)}[n-1+\delta(\lambda(n-\sigma)+1-\sigma)]}{(n-1) !} a_{n} \leq \delta(\lambda+1)(1-\sigma),
$$

where $0 \leq \lambda<1,0 \leq \sigma<1,0<\delta \leq 1,0 \leq \mu \leq 1$ and $z \in U$.

The result is sharp for the function $B_{\mu}$ given by

$$
B_{\mu}(z)=z-\frac{\delta(\lambda+1)(1-\sigma)(n-1) !}{n(\mu(n-1))^{n-2} e^{-\mu(n-1)}[n-1+\delta(\lambda(n-\sigma)+1-\sigma)]} z^{n}, \quad(n \geq 2) .
$$

Proof. Assume that the inequality (2.2) holds true $|z|=1$. Then, we obtain

$$
\left|z B_{\mu}^{\prime \prime}(z)\right|-\delta\left|\lambda z B_{\mu}^{\prime \prime}(z)+(\lambda+1)(1-\sigma) B_{\mu}^{\prime}(z)\right|
$$




$$
\begin{aligned}
= & \left|-\sum_{n=2}^{\infty} \frac{n(n-1)(\mu(n-1))^{n-2} e^{-\mu(n-1)}}{(n-1) !} a_{n} z^{n-1}\right| \\
& -\left|\delta(\lambda+1)(1-\sigma)-\sum_{n=2}^{\infty} \frac{\delta n(\lambda(n-\sigma)+1-\sigma)(\mu(n-1))^{n-2} e^{-\mu(n-1)}}{(n-1) !} a_{n} z^{n-1}\right| \\
\leq & \sum_{n=2}^{\infty} \frac{n(n-1)(\mu(n-1))^{n-2} e^{-\mu(n-1)}}{(n-1) !} a_{n}|z|^{n-1}-\delta(\lambda+1)(1-\sigma) \\
& +\sum_{n=2}^{\infty} \frac{\delta n(\lambda(n-\sigma)+1-\sigma)(\mu(n-1))^{n-2} e^{-\mu(n-1)}}{(n-1) !} a_{n}|z|^{n-1} \\
= & \sum_{n=2}^{\infty} \frac{n(\mu(n-1))^{n-2} e^{-\mu(n-1)}[n-1+\delta(\lambda(n-\sigma)+1-\sigma)]}{(n-1) !} a_{n}-\delta(\lambda+1)(1-\sigma) \leq 0,
\end{aligned}
$$

by hypothesis. Hence, by maximum modulus principle, we have $B_{\mu} \in \mathcal{H}(\lambda, \sigma, \delta, \mu)$.

Conversely, let $B_{\mu} \in \mathcal{H}(\lambda, \sigma, \delta, \mu)$. Then from (2.2), we obtain

$$
\begin{aligned}
& \left|\frac{z B_{\mu}^{\prime \prime}(z)}{\lambda z B_{\mu}^{\prime \prime}(z)+(\lambda+1)(1-\sigma) B_{\mu}^{\prime}(z)}\right| \\
= & \left|\frac{\sum_{n=2}^{\infty} \frac{n(n-1)(\mu(n-1))^{n-2} e^{-\mu(n-1)}}{(n-1) !} a_{n} z^{n-1}}{(\lambda+1)(1-\sigma)-\sum_{n=2}^{\infty} \frac{n(\lambda(n-\sigma)+1-\sigma)(\mu(n-1))^{n-2} e^{-\mu(n-1)}}{(n-1) !} a_{n} z^{n-1}}\right|<\delta .
\end{aligned}
$$

Since $\operatorname{Re}(z) \leq|z|$ for all $z(z \in U)$, we get

$$
\operatorname{Re}\left\{\frac{\sum_{n=2}^{\infty} \frac{n(n-1)(\mu(n-1))^{n-2} e^{-\mu(n-1)}}{(n-1) !} a_{n} z^{n-1}}{(\lambda+1)(1-\sigma)-\sum_{n=2}^{\infty} \frac{n(\lambda(n-\sigma)+1-\sigma)(\mu(n-1))^{n-2} e^{-\mu(n-1)}}{(n-1) !} a_{n} z^{n-1}}\right\}<\delta
$$


We choose the value of $z$ on the real axis so that $\frac{z B_{\mu}^{\prime \prime}(z)}{B_{\mu}^{\prime}(z)}$ is real. Upon clearing the denominator of (2.4) and letting $z \rightarrow 1^{-}$, through real values, thus we can write (2.4) as

$$
\sum_{n=2}^{\infty} \frac{n(\mu(n-1))^{n-2} e^{-\mu(n-1)}[n-1+\delta(\lambda(n-\sigma)+1-\sigma)]}{(n-1) !} a_{n} \leq \delta(\lambda+1)(1-\sigma),
$$

which completes the proof.

Corollary 2.1. If $B_{\mu} \in \mathcal{H}(\lambda, \sigma, \delta, \mu)$, then

$$
a_{n} \leq \frac{\delta(\lambda+1)(1-\sigma)(n-1) !}{n(\mu(n-1))^{n-2} e^{-\mu(n-1)}[n-1+\delta(\lambda(n-\sigma)+1-\sigma)]}, \quad(n \geq 2) .
$$

In the following theorem, we find integral representation of the family $\mathcal{H}(\lambda, \sigma, \delta, \mu)$.

Theorem 2.2. Let $B_{\mu} \in \mathcal{H}(\lambda, \sigma, \delta, \mu)$. Then

$$
B_{\mu}(z)=\int_{0}^{z} \exp \left[\int_{0}^{z} \frac{\eta(\lambda+1)(1-\sigma) \psi\left(t_{1}\right)}{t_{1}\left(1-\eta \lambda \psi\left(t_{1}\right)\right)} d t_{1}\right] d t_{2},
$$

where $|\psi(z)|<1, z \in U$.

Proof. By letting $\frac{z B_{\mu}^{\prime \prime}(z)}{B_{\mu}^{\prime}(z)}=E(z)$ in (2.1), we have

$$
\left|\frac{E(z)}{\lambda E(z)+(\lambda+1)(1-\sigma)}\right|<\delta
$$

or equivalently

$$
\frac{E(z)}{\lambda E(z)+(\lambda+1)(1-\sigma)}=\eta \psi(z), \quad(|\psi(z)|<1, z \in U) .
$$

So

$$
\frac{B_{\mu}^{\prime \prime}(z)}{B_{\mu}^{\prime}(z)}=\frac{\eta(\lambda+1)(1-\sigma) \psi(z)}{z(1-\eta \lambda \psi(z))}
$$


after integration, we obtain

$$
\log \left(B_{\mu}^{\prime}(z)\right)=\int_{0}^{z} \frac{\eta(\lambda+1)(1-\sigma) \psi(t)}{t(1-\eta \lambda \psi(t))} d t
$$

Therefore

$$
B_{\mu}^{\prime}(z)=\exp \left[\int_{0}^{z} \frac{\eta(\lambda+1)(1-\sigma) \psi(t)}{t(1-\eta \lambda \psi(t))} d t\right] .
$$

By integration once again, we get

$$
B_{\mu}(z)=\int_{0}^{z} \exp \left[\int_{0}^{z \eta} \frac{\eta(\lambda+1)(1-\sigma) \psi\left(t_{1}\right)}{t_{1}\left(1-\eta \lambda \psi\left(t_{1}\right)\right)} d t_{1}\right] d t_{2},
$$

and this gives the required result.

Theorem 2.3. If $B_{\mu} \in \mathcal{H}(\lambda, \sigma, \delta, \mu)$, then $B_{\mu}$ is starlike of order $\alpha(0 \leq \alpha<1)$ in the disk $|z|<r_{1}$, where

$$
r_{1}=\inf _{n}\left\{\frac{n(1-\alpha)[n-1+\delta(\lambda(n-\sigma)+1-\sigma)]}{\delta(n-\alpha)(\lambda+1)(1-\sigma)}\right\}^{\frac{1}{n-1}}, \quad(n \geq 2) .
$$

The result is sharp for the function $B_{\mu}$ given by (2.3).

Proof. It is sufficient to show that

$$
\left|\frac{z B_{\mu}^{\prime}(z)}{B_{\mu}(z)}-1\right| \leq 1-\alpha \text { for }|z|<r_{1} .
$$

We have

$$
\left|\frac{z B_{\mu}^{\prime}(z)}{B_{\mu}(z)}-1\right| \leq \frac{\sum_{n=2}^{\infty} \frac{(n-1)(\mu(n-1))^{n-2} e^{-\mu(n-1)}}{(n-1) !} a_{n}|z|^{n-1}}{1-\sum_{n=2}^{\infty} \frac{(\mu(n-1))^{n-2} e^{-\mu(n-1)}}{(n-1) !} a_{n}|z|^{n-1}} .
$$

Thus (2.5) will be satisfied if

$$
\sum_{n=2}^{\infty} \frac{(n-\alpha)(\mu(n-1))^{n-2} e^{-\mu(n-1)}}{(1-\alpha)(n-1) !} a_{n}|z|^{n-1} \leq 1 .
$$


Also from Theorem 2.1, if $B_{\mu} \in \mathcal{H}(\lambda, \sigma, \delta, \mu)$, then

$$
\sum_{n=2}^{\infty} \frac{n(\mu(n-1))^{n-2} e^{-\mu(n-1)}[n-1+\delta(\lambda(n-\sigma)+1-\sigma)]}{\delta(\lambda+1)(1-\sigma)(n-1) !} a_{n} \leq 1 .
$$

In view of (2.7), we notice that (2.6) holds true if

$$
\begin{aligned}
& \frac{(n-\alpha)(\mu(n-1))^{n-2} e^{-\mu(n-1)}}{(1-\alpha)(n-1) !}|z|^{n-1} \\
\leq & \frac{n(\mu(n-1))^{n-2} e^{-\mu(n-1)}[n-1+\delta(\lambda(n-\sigma)+1-\sigma)]}{\delta(\lambda+1)(1-\sigma)(n-1) !},
\end{aligned}
$$

or equivalently

$$
|z| \leq\left\{\frac{n(1-\alpha)[n-1+\delta(\lambda(n-\sigma)+1-\sigma)]}{\delta(n-\alpha)(\lambda+1)(1-\sigma)}\right\}^{\frac{1}{n-1}},
$$

this given the desired result.

Theorem 2.4. If $B_{\mu} \in \mathcal{H}(\lambda, \sigma, \delta, \mu)$, then $B_{\mu}$ is convex of order $\alpha(0 \leq \alpha<1)$ in the disk $|z|<r_{2}$, where

$$
r_{2} \leq \inf _{n}\left\{\frac{(1-\alpha)[n-1+\delta(\lambda(n-\sigma)+1-\sigma)]}{\delta(n-\alpha)(\lambda+1)(1-\sigma)}\right\}^{\frac{1}{n-1}}, \quad(n \geq 2) .
$$

The result is sharp for the function $B_{\mu}$ given by (2.3).

Proof. It is sufficient to show that

$$
\left|\frac{z B_{\mu}^{\prime \prime}(z)}{B_{\mu}^{\prime}(z)}\right| \leq 1-\alpha \text { for }|z|<r_{2}
$$

The result follows by application of arguments similar to the proof of Theorem 2.3.

Next, we obtain an extreme point of the family $B_{\mu} \in \mathcal{H}(\lambda, \sigma, \delta, \mu)$.

Theorem 2.5. Let $B_{\mu_{1}}(z)=z$ and 


$$
B_{\mu_{n}}(z)=z-\frac{\delta(\lambda+1)(1-\sigma)(n-1) !}{n(\mu(n-1))^{n-2} e^{-\mu(n-1)}[n-1+\delta(\lambda(n-\sigma)+1-\sigma)]} z^{n}, \quad(n \geq 2) .
$$

Then $B_{\mu} \in \mathcal{H}(\lambda, \sigma, \delta, \mu)$ if and only if it can be expressed in the form

$$
B_{\mu}(z)=\sum_{n=1}^{\infty} \gamma_{n} B_{\mu_{n}}(z)
$$

where $\gamma_{n} \geq 0, n \geq 1$ and $\sum_{n=1}^{\infty} \gamma_{n}=1$.

Proof. Suppose that $B_{\mu}$ is expressed in the form (2.8). Then

$$
\begin{aligned}
B_{\mu}(z)= & \sum_{n=1}^{\infty} \gamma_{n} B_{\mu_{n}}(z)=\gamma_{1} B_{\mu_{1}}(z)+\sum_{n=2}^{\infty} \gamma_{n} B_{\mu_{n}}(z) \\
= & \left(1-\sum_{n=2}^{\infty} \gamma_{n}\right) z \\
& +\sum_{n=2}^{\infty} \gamma_{n}\left(z-\frac{\delta(\lambda+1)(1-\sigma)(n-1) !}{n(\mu(n-1))^{n-2} e^{-\mu(n-1)}[n-1+\delta(\lambda(n-\sigma)+1-\sigma)]} z^{n}\right) \\
= & z-\sum_{n=2}^{\infty} \frac{\delta(\lambda+1)(1-\sigma)(n-1) !}{n(\mu(n-1))^{n-2} e^{-\mu(n-1)}[n-1+\delta(\lambda(n-\sigma)+1-\sigma)]} \gamma_{n} .
\end{aligned}
$$

Now

$$
\begin{aligned}
& \sum_{n=2}^{\infty} \frac{n(\mu(n-1))^{n-2} e^{-\mu(n-1)}[n-1+\delta(\lambda(n-\sigma)+1-\sigma)]}{\delta(\lambda+1)(1-\sigma)(n-1) !} \\
& \times \frac{\delta(\lambda+1)(1-\sigma)(n-1) !}{n(\mu(n-1))^{n-2} e^{-\mu(n-1)}[n-1+\delta(\lambda(n-\sigma)+1-\sigma)]} \gamma_{n} \\
= & \sum_{n=2}^{\infty} \gamma_{n}=1-\gamma_{1} \leq 1 .
\end{aligned}
$$

This shows that $B_{\mu} \in \mathcal{H}(\lambda, \sigma, \delta, \mu)$. 
Conversely, assume that $B_{\mu}$ given by (1.5) is in the family $\mathcal{H}(\lambda, \sigma, \delta, \mu)$. Then by Corollary 2.1, we find that

$$
a_{n} \leq \frac{\delta(\lambda+1)(1-\sigma)(n-1) !}{n(\mu(n-1))^{n-2} e^{-\mu(n-1)}[n-1+\delta(\lambda(n-\sigma)+1-\sigma)]},
$$

we can set

$$
\gamma_{n}=\frac{n(\mu(n-1))^{2(n-2)} e^{-2 \mu(n-1)}[n-1+\delta(\lambda(n-\sigma)+1-\sigma)]}{\delta(\lambda+1)(1-\sigma)((n-1) !)^{2}} a_{n}, \quad(n \geq 2),
$$

where $\gamma_{1}=1-\sum_{n=2}^{\infty} \gamma_{n}$. Thus

$$
\begin{aligned}
B_{\mu}(z)= & z-\sum_{n=2}^{\infty} \frac{(\mu(n-1))^{n-2} e^{-\mu(n-1)}}{(n-1) !} a_{n} z^{n} \\
= & z-\sum_{n=2}^{\infty} \frac{(\mu(n-1))^{n-2} e^{-\mu(n-1)}}{(n-1) !} \\
& \times \frac{\delta(\lambda+1)(1-\sigma)((n-1) !)^{2}}{n(\mu(n-1))^{2(n-2)} e^{-2 \mu(n-1)}[n-1+\delta(\lambda(n-\sigma)+1-\sigma)]} \gamma_{n} z^{n} \\
= & z-\sum_{n=2}^{\infty} \frac{\delta(\lambda+1)(1-\sigma)(n-1) !}{n(\mu(n-1))^{n-2} e^{-\mu(n-1)}[n-1+\delta(\lambda(n-\sigma)+1-\sigma)]} \gamma_{n} z^{n} \\
= & z-\sum_{n=2}^{\infty}\left(z-B_{\mu_{n}}(z)\right) \gamma_{n}=\left(1-\sum_{n=2}^{\infty} \gamma_{n}\right) z+\sum_{n=2}^{\infty} \gamma_{n} B_{\mu_{n}}(z) \\
= & \gamma_{1} B_{\mu_{1}}(z)+\sum_{n=2}^{\infty} \gamma_{n} B_{\mu_{n}}(z)=\sum_{n=1}^{\infty} \gamma_{n} B_{\mu_{n}}(z),
\end{aligned}
$$

that is the required representation.

In view of the work of Goodman [3] and Ruscheweyh [6], we define the $\tau$ neighborhood for the power series $B_{\mu}$ by means of the definition below: 


$$
\begin{aligned}
& N_{\tau}\left(B_{\mu}\right)=\left\{h_{\mu}: h_{\mu}(z)=z-\sum_{n=2}^{\infty} \frac{(\mu(n-1))^{n-2} e^{-\mu(n-1)}}{(n-1) !} b_{n} z^{n}\right. \\
& \left.\quad \text { and } \sum_{n=2}^{\infty} \frac{n(\mu(n-1))^{n-2} e^{-\mu(n-1)}}{(n-1) !}\left|a_{n}-b_{n}\right| \leq \tau, 0 \leq \tau<1\right\} .
\end{aligned}
$$

In particular, for the identity function $e(z)=z$, we have

$$
\begin{aligned}
N_{\tau}\left(e_{\mu}\right)= & \left\{h_{\mu}: h_{\mu}(z)=z-\sum_{n=2}^{\infty} \frac{(\mu(n-1))^{n-2} e^{-\mu(n-1)}}{(n-1) !} b_{n} z^{n}\right. \\
& \text { and } \left.\sum_{n=2}^{\infty} \frac{n(\mu(n-1))^{n-2} e^{-\mu(n-1)}}{(n-1) !}\left|b_{n}\right| \leq \tau, 0 \leq \tau<1\right\} .
\end{aligned}
$$

Definition 2.2. A function $B_{\mu}$ is said to be in the family $\mathcal{H}_{y}(\lambda, \sigma, \delta, \mu)$ if there exists a function $h_{\mu} \in \mathcal{H}(\lambda, \sigma, \delta, \mu)$, such that

$$
\left|\frac{B_{\mu}(z)}{h_{\mu}(z)}-1\right|<1-y \quad(z \in U, 0 \leq y<1) .
$$

Theorem 2.6. If $h_{\mu} \in H(\lambda, \sigma, \delta, \mu)$ and

$$
y=1-\frac{\tau[\delta(\lambda(2-\sigma)+1-\sigma)]}{2[\delta(\lambda(2-\sigma)+1-\sigma)]-\delta(\lambda+1)(1-\sigma)},
$$

then $N_{\tau}\left(h_{\mu}\right) \subset H_{y}(\lambda, \sigma, \delta, \mu)$.

Proof. Let $B_{\mu} \in N_{\tau}\left(h_{\mu}\right)$. Then we find from (2.9) that

$$
\sum_{n=2}^{\infty} \frac{n(\mu(n-1))^{n-2} e^{-\mu(n-1)}}{(n-1) !}\left|a_{n}-b_{n}\right| \leq \tau,
$$

which implies the coefficient inequality

$$
\sum_{n=2}^{\infty} \frac{(\mu(n-1))^{n-2} e^{-\mu(n-1)}}{(n-1) !}\left|a_{n}-b_{n}\right| \leq \frac{\tau}{2} .
$$


Since $h_{\mu} \in \mathcal{H}(\lambda, \sigma, \delta, \mu)$, then by using Theorem 2.1, we have

$$
\sum_{n=2}^{\infty} \frac{(\mu(n-1))^{n-2} e^{-\mu(n-1)}}{(n-1) !} b_{n} \leq \frac{\delta(\lambda+1)(1-\sigma)}{2[\delta(\lambda(2-\sigma)+1-\sigma)]} .
$$

So that

$$
\begin{aligned}
\left|\frac{B_{\mu}(z)}{h_{\mu}(z)}-1\right| \leq & \frac{\sum_{n=2}^{\infty} \frac{(\mu(n-1))^{n-2} e^{-\mu(n-1)}}{(n-1) !}\left|a_{n}-b_{n}\right||z|^{n-1}}{1-\sum_{n=2}^{\infty} \frac{(\mu(n-1))^{n-2} e^{-\mu(n-1)}}{(n-1) !} b_{n}|z|^{n-1}} \\
& <\frac{\sum_{n=2}^{\infty} \frac{(\mu(n-1))^{n-2} e^{-\mu(n-1)}}{(n-1) !}\left|a_{n}-b_{n}\right|}{1-\sum_{n=2}^{\infty} \frac{(\mu(n-1))^{n-2} e^{-\mu(n-1)}}{(n-1) !} b_{n}} \\
& \leq \frac{\tau[\delta(\lambda(2-\sigma)+1-\sigma)]}{2[\delta(\lambda(2-\sigma)+1-\sigma)]-\delta(\lambda+1)(1-\sigma)}=1-y .
\end{aligned}
$$

Hence, by Definition 2.2, equivalently to $B_{\mu} \in \mathcal{H}_{y}(\lambda, \sigma, \delta, \mu)$ for $y$ given by (2.11). This completes the proof of the theorem.

\section{References}

[1] S. Altinkaya and S. Yalçin, Poisson distribution series for certain subclasses of starlike functions with negative coefficients, An. Univ. Oradea Fasc. Mat. 24(2) (2017), 5-8.

[2] S. M. El-Deeb, T. Bulboaca and J. Dziok, Pascal distribution series connected with certain subclasses of univalent functions, Kyungpook Math. J. 59(2) (2019), 301-314.

[3] A. W. Goodman, Univalent functions and non-analytic curves, Proc. Amer. Math. Soc. 8 (1975), 598-601. https://doi.org/10.1090/S0002-9939-1957-0086879-9

[4] W. Nazeer, Q. Mehmood, S. M. Kang and A. Ul Haq, An application of binomial distribution series on certain analytic functions, J. Comput. Anal. Appl. 26 (2019), 11-17.

[5] S. Porwal and M. Kumar, A unified study on starlike and convex functions associated with Poisson distribution series, Afr. Mat. 27 (2016), 10-21. https://doi.org/10.1007/s13370-016-0398-z 
[6] S. Ruscheweyh, Neighborhoods of univalent functions, Proc. Amer. Math. Soc. 81 (1981), 521-527. https://doi.org/10.1090/S0002-9939-1981-0601721-6

[7] H. Silverman, Univalent functions with negative coefficients, Proc. Amer. Math. Soc. 51(1) (1975), 109-116. https://doi.org/10.1090/S0002-9939-1975-0369678-0 